MATEC Web of Conferences 48, 04003 (2016)

DOI: $10.1051 /$ matecconf $/ 20164804003$

(C) Owned by the authors, published by EDP Sciences, 2016

\title{
Speckle pattern processing by digital image correlation
}

\author{
Fedor Gubarev ${ }^{1,2, a}$, Lin $\mathrm{Li}^{1}$, Miron Klenovskii ${ }^{1,3}$ and Anatoliy Glotov ${ }^{1}$ \\ ${ }^{1}$ National Research Tomsk Polytechnic University, 634050 Tomsk, Russia
}

\begin{abstract}
Testing the method of speckle pattern processing based on the digital image correlation is carried out in the current work. Three the most widely used formulas of the correlation coefficient are tested. To determine the accuracy of the speckle pattern processing, test speckle patterns with known displacement are used. The optimal size of a speckle pattern template used for determination of correlation and corresponding the speckle pattern displacement is also considered in the work.
\end{abstract}

\section{Introduction}

The progress of space exploration and space technologies is inseparably linked with the development of new methods and means of remote obtaining information about the objects and processes. The objects under control can be living organisms as well as various machine parts during the process of their manufacturing or their operation. Promising technics of remote control and at the same time nondestructive testing are the optical methods, and specifically methods based on the use of coherent radiation [1-6].

One of the most promising technics for the measurement of microdisplacements and deformations is a method of digital speckle correlation [6-8]. This method, which has found wide application in nondestructive testing of materials [9-11], is also a promising method of diagnostics in biology and medicine [7, 12-15]. The method of digital speckle correlation is also suitable for the remote measurement of microdisplacements and speed of various processes [15-17]. In [15] the authors realized audio signal extraction from vibrating objects located at a distance up to $100 \mathrm{~m}$ by means of the digital speckle correlation method. This fact points to the great prospects of the technique. For instance, in a case of radio communication failure this feature can allow to realize a possibility to hear an astronaut when he/she is outside a space vehicle just directing a laser beam on the spacesuit.

The essence of the digital speckle correlation technique consists in illuminating the object under investigation by coherent radiation with simultaneous recording speckle patterns formed by light scattered from the object and subsequent their processing by means of the method of digital image correlation. In [15] is shown that when optical system being used is properly configured, the displacement of an object under observation results in proportional transverse shift of the formed speckle pattern. This fact allows to detect the displacement as well as vibration of the object. The displacement of objects under control can be caused by various factors which can be both desirable and undesirable. Having established a correlation between the change in the formed speckle pattern

${ }^{a}$ Corresponding author : gubarevfa@tpu.ru

${ }^{2}$ V.E. Zuev Institute of Atmospheric Optics, Russian Academy of Sciences, 634021 Tomsk, Russia

${ }^{3}$ Institute of Electron Physics, National Academy of Sciences of Ukraine, 88017 Uzhgorod, Ukraine 
and the change in a controlled process, it is possible to realize a complete control of the process by implementing the appropriate control action (a feedback).

One of the main requirements for the method is the high reliability and accuracy of determining the displacement of an object of interest. The aim of the current research is to study the method of speckle pattern processing by digital image correlation. The optimal size of a speckle pattern template used for determination of correlation and corresponding the object displacement is also investigated in the work.

\section{Description of speckle pattern processing}

When a rough surface of an object is illuminated by a coherent radiation, the beams reflected from the surface interfere and as a result spatially distributed speckle patterns are formed. Any displacement of an object under observation causes corresponding change in the spatial distribution of obtained speckle patterns. By establishing these changes one can determine the displacement of the object surface. Moreover, determining the time-resolved displacement of the object surface one can measure the vibration of the surface [18].

The determination of a surface displacement can be easily carried out by analysis of speckle pattern shift by means of digital image correlation. Let us consider the bottom lines of the method. The speckle patterns recorded before and after displacement of the surface are needed to carry out the analysis. The recorded digital speckle images are converted into matrices contained values of pixels intensity.

Figure 1 illustrates the change in position of a speckle after displacement. As one can see initially the position of a speckle has coordinates $\left(\mathrm{x}_{1}, \mathrm{y}_{1}\right)$ but after displacement it changes to the $\left(\mathrm{x}_{2}, \mathrm{y}_{2}\right)$. Let the intensity in the some part of the speckle pattern before the displacement is $f(\mathrm{x}, \mathrm{y})$ while its intensity after the displacement is $g(\mathrm{x}, \mathrm{y})$. If in the image obtained after displacement one can find a region $g$ correlated best with the initial speckle $f$, then one can conclude that speckle is displaced from the position of $f$ to the position of $g$.

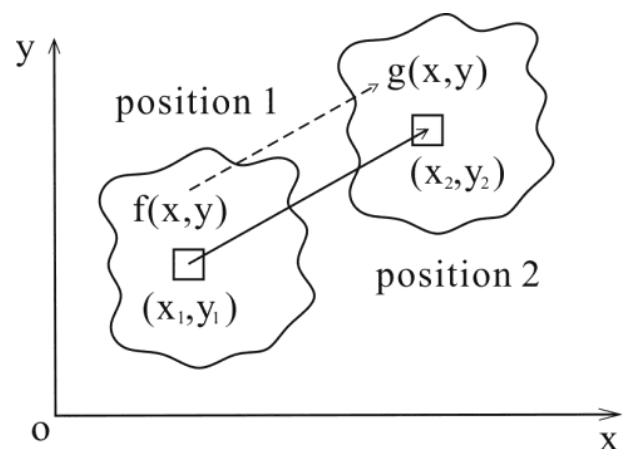

Figure 1. Changing a speckle position due to displacement.

To find the position of $g$ it has been realized an algorithm in the MATLAB software environment. The main idea of the algorithm consists in finding the location of the correlation coefficient maximum. The algorithm contains the following three basic steps.

1. In the first image a part of the speckle pattern (hereinafter referred to as template) is chosen which then is searched in the next images. If the chosen template is of too small size, it is impossible to localize its position in the next images because of lack of unique information in the template of small size. If the chosen template is of too big size, it takes a lot of time for the processing of images. In [8] is mentioned that the optimal size of the template is found over the range $31 \times 31$ pixels to $61 \times 61$ pixels. However, no detailed explanations regarding this conclusion are given. Therefore, in the current work the special attention is paid to the question about finding the optimal size of the template.

2. The algebraical expression for calculating the correlation coefficients is chosen. The most widely used formulas of the correlation coefficient are the following [9, 10, 19-22]: 


$$
\begin{gathered}
C_{1}=\frac{\sum_{i=1}^{n} \sum_{j=1}^{m}\left[f\left(x_{i}, y_{i}\right) \cdot g\left(x_{i}, y_{i}\right)\right]}{\sqrt{\sum_{i=1}^{n} \sum_{j=1}^{m} f^{2}\left(x_{i}, y_{i}\right) \cdot \sum_{i=1}^{n} \sum_{j=1}^{m} g^{2}\left(x_{i}, y_{i}\right)}} \\
C_{2}=\frac{\sum_{i=1}^{n} \sum_{j=1}^{m}\left[f\left(x_{i}, y_{i}\right)-\bar{f}\right] \cdot\left[g\left(x_{i}, y_{i}\right)-\bar{g}\right]}{\sqrt{\sum_{i=1}^{n} \sum_{j=1}^{m}\left[f\left(x_{i}, y_{i}\right)-\bar{f}\right]^{2} \cdot \sum_{i=1}^{n} \sum_{j=1}^{m}\left[g\left(x_{i}, y_{i}\right)-\bar{g}\right]^{2}}} \\
C_{3}=\left(C_{2}\right)^{2}=\frac{\left\{\sum_{i=1}^{n} \sum_{j=1}^{m}\left[f\left(x_{i}, y_{i}\right)-\bar{f}\right] \cdot\left[g\left(x_{i}, y_{i}\right)-\bar{g}\right]\right\}^{2}}{\sum_{i=1}^{n} \sum_{j=1}^{m}\left[f\left(x_{i}, y_{i}\right)-\bar{f}\right]^{2} \cdot \sum_{i=1}^{n} \sum_{j=1}^{m}\left[g\left(x_{i}, y_{i}\right)-\bar{g}\right]^{2}}
\end{gathered}
$$

Where, $\bar{f}$ is the average intensity of the reference (initial) speckle pattern (template); $f\left(x_{i}, y_{i}\right)$ is intensity of its pixel with coordinates $\left(x_{i}, y_{i}\right) ; \bar{g}$ is the average intensity of the speckle pattern obtained after displacement; $g\left(x_{i}, y_{i}\right)$ is intensity of its pixel with coordinates $\left(x_{i}, y_{i}\right)$.

The main requirements for the formula of the correlation coefficient calculation are reliability, noise immunity, and speed of calculating. In practice, the formula (2) is the most widely used. It should be mentioned that in this step the location of the correlation coefficient maximum is found with accuracy within $+/-0.5$ pixels.

3. The correlation coefficients around the found maximum value are chosen to carry out their interpolation with a step of 0.01 pixels or even less. Thus, a more accurate location of the correlation coefficient maximum is found.

\section{Testing the method of speckle pattern correlation}

In the current work testing of the realized algorithm is carried out by means of the test speckle patterns with known displacement. In [23] a method for producing high-precision experimentally subpixel shifted images has been created. The author [23] produced a set of speckle patterns shifted with a step of 0.1 pixels. There are available two sets of speckle patterns with high and low contrast [24] which are used in the current work for determining the accuracy of the algorithm. Figure 2 exhibits the trajectory of speckle pattern shifting and its determination by the algorithm under testing.
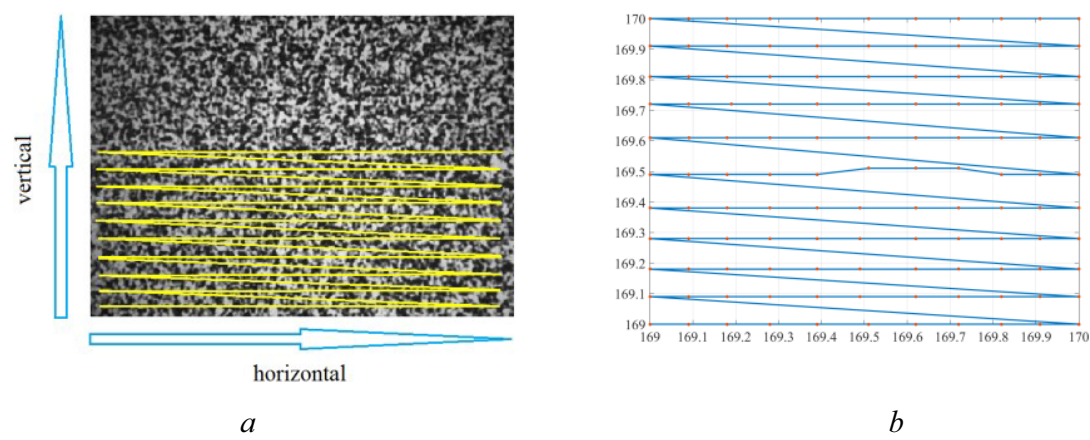

Figure 2. A speckle pattern and the trajectory (yellow curve) of its shifting (a) and the trajectory of the speckle pattern displacement determined by the algorithm under testing $(b)$. 

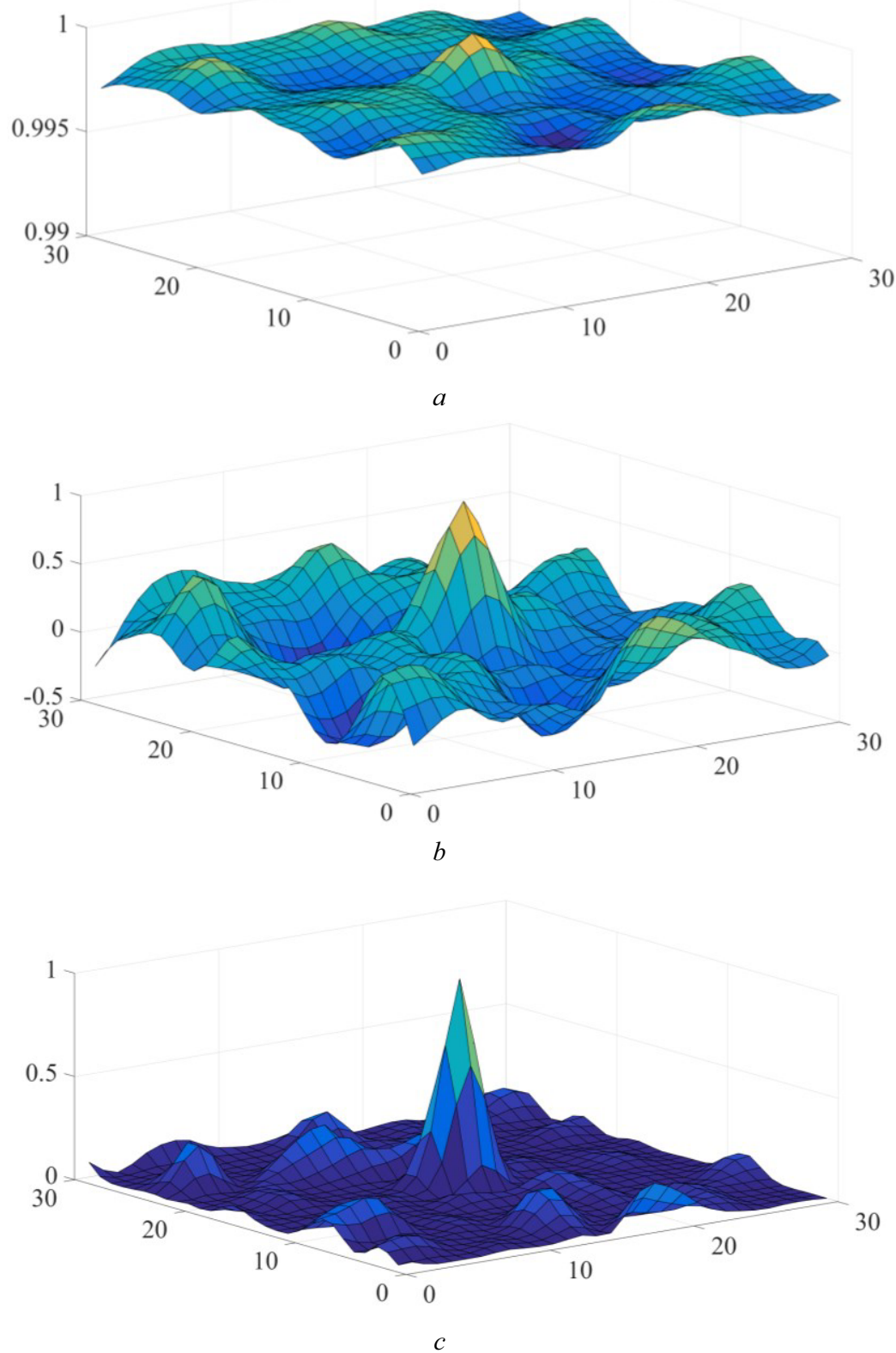

Figure 3. Distribution of the correlation coefficient calculated by the formulas: a) - (1); b) - (2); c) - (3).

Figure 3 presents distribution of the correlation coefficient calculated by the formulas $C_{1}-C_{3}$ for the high contrast speckle pattern borrowed from [24]. One can notice that the distribution of the correlation coefficient calculated by the formula (1) is not characterized by unimodality and clear peak. As a result the formula (1) cannot ensure the reliable localization of the correlation coefficient maximum. The calculation carried out by the formula (2) gives a lot of peaks but the main peak is already stood out well against the others. The calculation performed by the formula (3) results in the best unimodality. The difference between the main peak and the others is noticeably more. However, the question about the formula ensured the best final result is not obvious. To clear up the question the test calculations were carried out. 


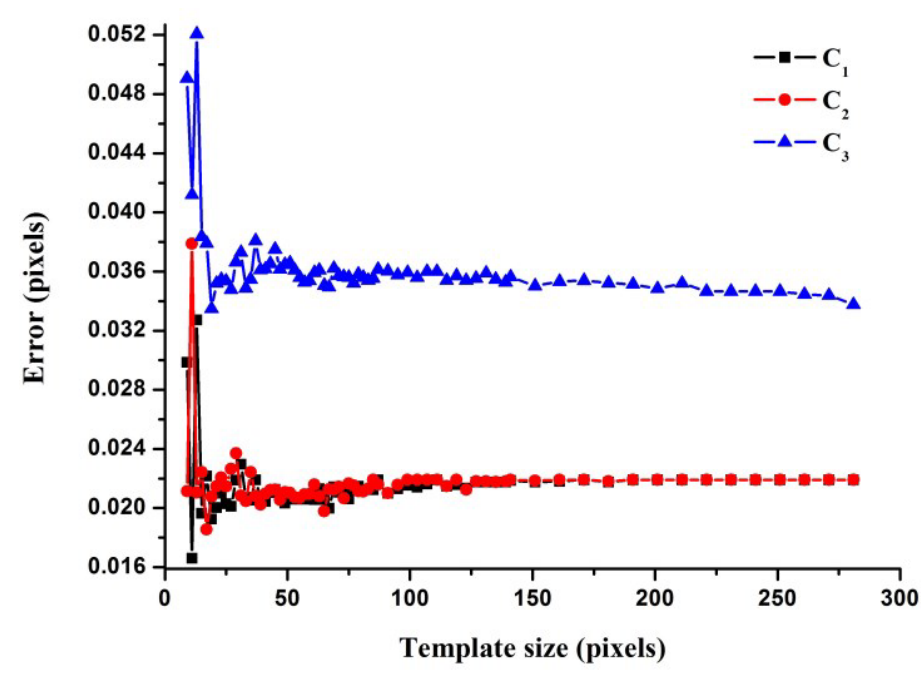

$a$

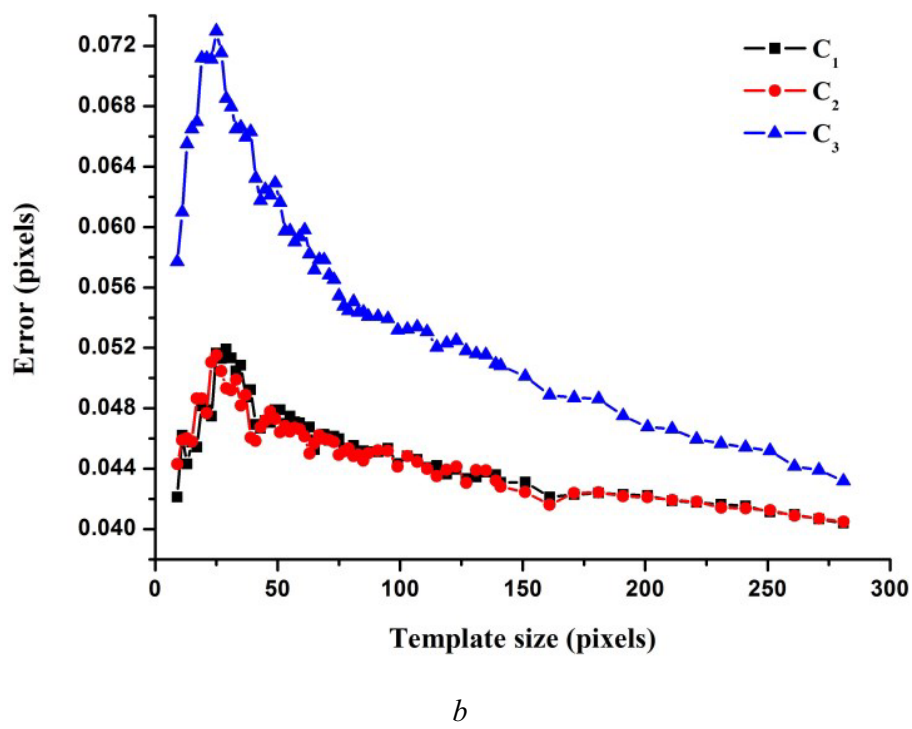

Figure 4. Dependence of the average absolute error of displacement determination on the template size (side length) for the different formulas of correlation coefficient.

Figure 4 shows dependence of the average absolute error of displacement determination on the template size for the different formulas of correlation coefficient. The calculation by the formulas (1) and (2) gives almost the same rusults (figure 4). The average absolute error of displacement determination by the formulas (1) and (2) amounted to 0.022 pixels for the high contrast speckle pattern set and 0.044 pixels for the low contrast speckle pattern set. As one can see the calculation by the formula (3) gives almost twice increase in the error of displacement determination dispite the presence of clear main peak (figure 3c). One can suppose that this is due to increasing the difference in the correlation coefficients around the main peak which leads to deterioration of the interpolation quality and as a result to the accuracy degradation of the correlation maximum localization.

It should be also noted that when the template size is less than $30 \times 30$ pixels the accuracy of displacement determination is not stable. To get the reliable results the template size should be more than $30 \times 30$ pixels. In the case of low contrast speckle patterns the bigger template size gives the better accuracy (figure 4b). 


\section{Conclusion}

Studying the method of speckle pattern processing based on the digital image correlation was carried out. The three formulas of correlation coefficients were investigated. The first two formulas were turned out to give the best accuracy of determining the speckle pattern displacement. The formulas ensured the average absolute error of displacement determination about 0.022 pixels for the high contrast speckle pattern set and 0.044 pixels for the low contrast speckle pattern set. However, there is a suspicion concerning the reliability of the first formula. One can suppose that in a case of speckle patterns with a noise the first formula can give unreliable results because of low noise immunity.

The optimal size of a speckle pattern template needed for determination of correlation and corresponding the speckle pattern displacement was also investigated in the work. When the template size is less than $30 \times 30$ pixels the accuracy of displacement determination was turned out to be not stable. To get the reliable results the template size should be more than $30 \times 30$ pixels.

\section{References}

1. I.P. Sukharev, I.P. Sukharev, Experimental Methods for the Study of Deformation and Strength (Mechanical Engineering, Moscow, 1987)

2. G.S. Evtushenko, D.V. Shiyanov, F.A. Gubarev, Metal Vapor Lasers with High Pulse Repetition Rates (Tomsk Polytechnic University Publishing House, Tomsk, 2010)

3. F.A. Gubarev, M.S. Klenovskii, Lin Li, IOP Conf. Series: Materials Science and Engineering 81, $012116(2015)$

4. F.A. Gubarev, M.V. Trigub, M.S. Klenovskii, Lin Li, G.S. Evtushenko, Appl. Phys. B. 122, 1 (2016)

5. M. Locatelli, E. Pugliese, M. Paturzo, V. Bianco, A. Finizio, A. Pelagotti, P. Poggi, L. Miccio, R. Meucci, P. Ferraro, Opt. Express 21, 5 (2013)

6. J.W. Goodman, Speckle Phenomena in Optics: Theory and Applications (Roberts and Company Publishers, Englewood, 2007)

7. V.V. Tuchin, Lasers and Fiber Optics in Biomedical Research. 2 edition. (Fizmatlit, Moscow, 2010)

8. G. Jin, L. Meng, J. Chen, S. Ma, J. Zhang, Exp. Mech. 21, 689 (2006) (in Chinese)

9. D. Zhang, X. Zhang, G. Cheng, Exp. Mech. 39, 62 (1999)

10. H. Lu, P.D. Cary, Exp. Mech. 40, 393 (2000)

11. J. Gonzalez, W.G. Knauss, J. Mech. Phys. Solids 46, 1981 (1998)

12. V.V. Tuchin, J. Biomed. Opt. 4, 106 (1999)

13. M.A. Vilensky, D.N. Agafonov, D.A. Zimnyakov, V.V. Tuchin, R.A. Zdrazhevskii, Quantum Electron 41, 324 (2011)

14. Y. Beiderman, N. Burshtein, M. Teicher, V. Mico, Z. Zalevsky, J. Biomed. Opt. 15, 061707 (2010)

15. Z. Zalevsky, Y. Beiderman, I. Margalit, S. Gingold, M. Teicher, V. Mico, J. Garcia, Opt. Express 17, 21566 (2009)

16. B.R. Durig, W.H. Peters, M.A. Hammer, Proc. SPIE 0954, 438 (1989)

17. N.A. Russo, J.A. Pomarico, E.E. Sicre, D. Patrignani, L. Pasquale, Opt. Commun. 169, 23 (1999)

18. Lin Li, F.A. Gubarev, M.S. Klenovskii, A.I. Bloshkina, International Siberian Conference on Control and Communications (SIBCON) (to be published)

19. K. Okamoto, Y.A. Hassan, W.D. Schmidi, Exp. Fluids 19, 342 (1995)

20. T. Roesgen, R. Totaro, Exp. Fluids 19, 188 (1995)

21. L. Gui, W. Merzkirch, Exp. Fluids 28, 36 (2000)

22. W.S. Schreier, J.R. Braasch, M.A. Sutton, Opt. Eng. 39, 2915 (2000)

23. P.L. Reu, Exp. Mech. 51, 443 (2011)

24. Digital image correlation challenge. URL: http://www.sem.org/dic-challenge (2016) 